\title{
Energy Estimation Based on Path Tracking for a Differential Drive Wheeled Mobile Robot
}

\author{
Said Fadlo*, Nabila Rabbah, and Abdelhafid Ait Elmahjoub \\ Laboratory of Structural Engineering, Intelligent Systems and Electrical Energy, Department of Electrical Engineering, \\ Hassan II University, Casablanca, Morocco
}

\begin{abstract}
To improve the energy efficiency of mobile robots and increase their time of operation, a comprehensive energy model is needed. Having such a model requires a lot of complex analysis and design time. There has been a lot of research into optimizing the power consumption of mobile robots but have not benefited from the advantages of languages to model complex cyber-physical systems. In this work, we used the Simscape ${ }^{\mathrm{TM}}$ MATLAB $^{\circledR}$ environment to simplify and speed up the design of an energy consumption model of a differential drive mobile robot. We also estimated the energy consumption of the mobile in a different path tracking scenario. Our results show that is possible to obtain a good accuracy of path following with acceptable energy consumption.
\end{abstract}

\section{Introduction}

Due to immense progress in the field of robotics, mobile robot can now perform a lot of complicated tasks. That is achieved by using a battery as the main power source. Though, batteries have a very limited energy capacity. The above-mentioned problem can be solved by increasing battery life or reducing energy consumption. Many researchers have been focused on designing motion planning techniques that can reduce the energy consumption of mobile robot. Kim and Kim [1-3] derived an energy equation for a differential drive robot in terms of robot's velocity vector. The equation was then optimized, and velocity profiles were generated for the robot which helped them save $10 \%$ more energy compared to the famous trapezoidal velocity profile. Their energy model was, however, not complete because they did not consider the power consumption in the electronics of the robot. Despite the researcher's efforts to obtain better trajectories [5-7], the resulting energy values did not reflect the contribution of mobile dynamic parameters, such as the weight of the mobile or load weight.

J.Morales et.al [8], presented a mixed energy model that considers the DC motor and the mobile robot dynamic, but did not take into consideration losses due to rolling friction and losses in controller electronics .

To analyze and manage all the problems with energy consumption and robot stability, a complete approach of robot system design considering multiphysics of mechanics, electrics, aerodynamics, control, and so on is necessary. The Simscape ${ }^{\mathrm{TM}}$ MATLAB $^{\circledR}$ environment enables physical modeling, i.e. to model the physical components and their interconnections. The tool also finds the equations and solve them.
In this paper, we used MathWorks ${ }^{\circledR}$ simulation tool MATLAB $^{\circledR}$ Simscape $^{\mathrm{TM}}$ environment to simplify and speed up the design of an energy consumption model of a mobile robot. We also studied the different components of energy consumption in a differential drive robot and presented an energy model. In order to validate our model, we compared it to the mixed energy model and the DC motor model.

This paper is organized as follows: Section II presents the energy model. Section III describes the implementation of the model in Simscape ${ }^{\mathrm{TM}}$ environment, and the results simulation of the energy model. Finally, conclusions are presented in Section IV.

\section{System Modeling}

\subsection{Kinematic model}

To develop an energy model of a differential mobile, it important to present its kinematics model. A differential robot has two driven wheels which are attached to the DC motors and a castor which is added for balancing. A kinematic model is shown in Figure 1. $\dot{\theta}_{L}$ and $\dot{\theta}_{R}$ are the rotational velocities of the DC motors which are related to the linear $v$ and rotational $\omega$ velocities of the robot by the following equations:

$$
\begin{aligned}
& v=\frac{r\left(\dot{\theta}_{R}+\dot{\theta}_{L}\right)}{2} \\
& \omega=\frac{r\left(\dot{\theta}_{R}-\dot{\theta}_{L}\right)}{2 l}
\end{aligned}
$$

\footnotetext{
* Corresponding author: fadlosaid@gmail.com
} 
The radius' of wheels are equal and symbolized by $r, l$ is the displacement from each of the driving wheels to the axis of symmetry as shown in Figure 1.

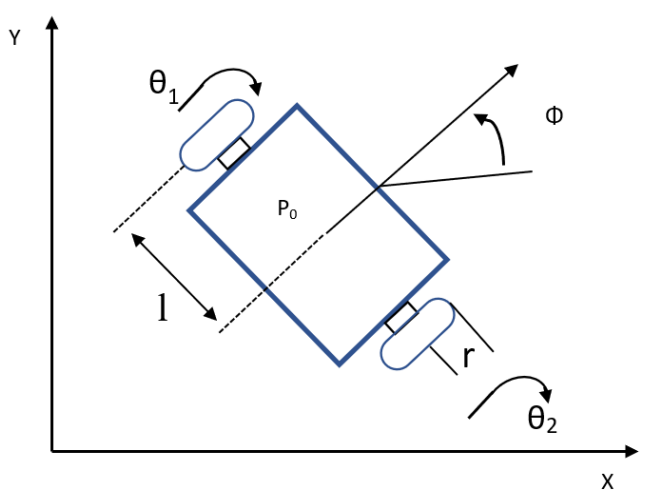

Fig. 1. Schematic of the differential mobile robot.

\subsection{DC motor Model}

The DC motor behaviour is characterized using an equivalent circuit model. Figure 2 shows the DC motor circuit with Torque and Rotor angle consideration. The differential equations that describe the dynamic model are:

$$
\left\{\begin{array}{c}
L \frac{d i}{d t}+R i+K_{\omega} \dot{\theta}=V \\
I_{S} \ddot{\theta}-K_{t} i+v \dot{\theta}+\tau=0
\end{array}\right.
$$

Where:

$\mathrm{V}$ and $\mathrm{i}$ are the armature voltage and current.

$\mathrm{R}$ and $\mathrm{L}$ are the armature resistance and inductance. $v$ is the viscous friction coefficient.

$\tau$ is the dynamic load applied to the motor.

$K_{t}$ is the motor torque constant.

$K_{\omega}$ is the voltage constant.

$I_{S}$ is the motor shaft inertia.

$\theta=\left[\theta_{1} \theta_{2}\right]$ are the angular positions of the wheels.

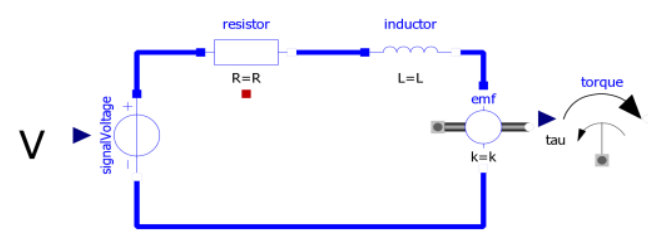

Fig. 2. Schematic of the DC Motor

\subsection{Mixed energy Model}

The system of nonlinear differential equations that represents the dynamic mobile model [10], can be determined by the Lagranges movement equation:

$$
\frac{d}{d t}\left(\frac{\partial T}{\partial \dot{q}_{l}}\right)-\left(\frac{\partial T}{\partial q}\right)=\tau_{i}-a_{1 i} a \lambda_{1}-a_{2 i} a \lambda_{2}
$$

$\mathrm{T}$ is the equation for mobile kinematic energy. $q=\left[x, y, \theta_{1}, \theta_{2}\right]^{T}$ is the vector of the state variables. $\lambda$ is the Lagrange's multipliers.

$\tau$ is the torques applied to the wheels.

$a_{i j}$ are the matrix movement restriction components.

The kinetic energy and the restriction equation matrix are replaced in Lagranges movement equation. Then $\tau$ placed into dynamic mobile model lading to a system of equations ordered and written in the following matrix, obtaining the mixed dynamic model [9] :

Where:

$$
M(q) \ddot{q}-F \dot{q}+C(q, \dot{q})=T V-\lambda A(q)
$$

$$
M(q)=\left[\begin{array}{cccc}
m & 0 & -\alpha_{1} & \alpha_{1} \\
0 & m & \alpha_{2} & -\alpha_{2} \\
-\alpha_{1} & \alpha_{2} & I & -I_{c}^{2} \\
\alpha_{1} & \alpha_{2} & -I_{c}^{2} & I
\end{array}\right]
$$

$$
\begin{aligned}
& \alpha_{1}=m_{c} c d \sin \phi \\
& \alpha_{2}=m_{c} c d \cos \phi \\
& I=I_{c}^{2}-I_{w}-I_{s}
\end{aligned}
$$

$$
F=\left[\begin{array}{cccc}
0 & 0 & 0 & 0 \\
0 & 0 & 0 & 0 \\
0 & 0 & \frac{K_{t} K_{w}}{R}+v & 0 \\
0 & 0 & 0 & \frac{K_{t} K_{w}}{R}+v
\end{array}\right]
$$

$$
\begin{aligned}
C(q, \dot{q}) & =\left[\begin{array}{cc}
-m_{c} c d \dot{\phi}^{2} & \cos \phi \\
-m_{c} c d \dot{\phi}^{2} & \sin \phi \\
0 & 0
\end{array}\right] \\
T & =\left[\begin{array}{cc}
0 & 0 \\
0 & 0 \\
\frac{K_{t}}{R} & 0 \\
0 & \frac{K_{t}}{R}
\end{array}\right] \\
A(q) & =\left[\begin{array}{cc}
-\sin \phi & -\cos \phi \\
\cos \phi & -\sin \phi \\
0 & c l \\
0 & c l
\end{array}\right]
\end{aligned}
$$

Where :

$m_{c}$ is the mass of the mobile robot.

$d$ is the displacement from $\mathrm{P}_{0}$ to the mass centre.

$c$ is a constant equal to $\frac{r}{2 l}$.

$I_{c}$ is the moment of inertia of the mobile robot without the driving wheels and the roto of the motors about vertical axis through the intersection of the axis of symmetry with the driving wheel axis.

$I_{w}$ is the moment of inertia of each driving wheel and the motor rotor about the wheel axis.

$I_{m}$ is the moment of inertia of each driving wheel and the motor rotor about the wheel diameter.

With $\mathrm{i}=1 \ldots 4$

Where: 


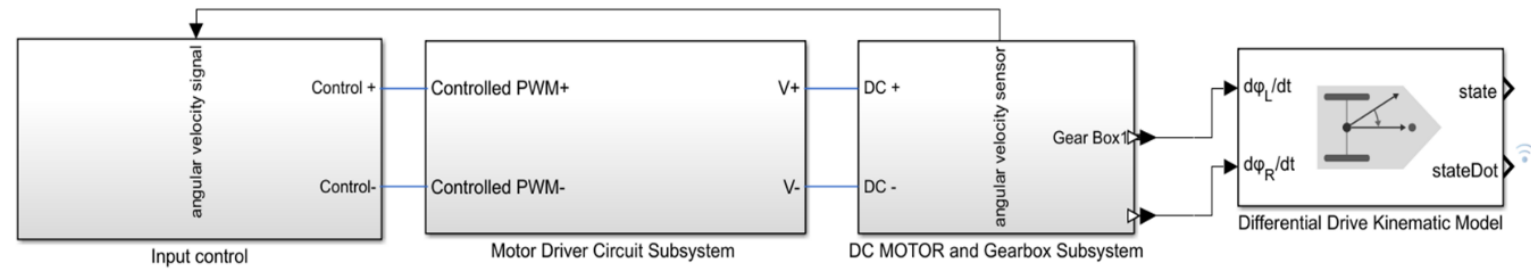

Fig. 3. Block diagram of the differential mobile robot

Table 1: Motor parameter of the WMR P3-DX.

\begin{tabular}{|c|c|}
\hline Parameters & Values \\
\hline$R$ & $0.7 \Omega$ \\
\hline$K_{t}$ & $0.88 \mathrm{Nm} / \mathrm{A}$ \\
\hline$K_{\omega}$ & $0.88(\mathrm{rad} / \mathrm{s}) / \mathrm{V}$ \\
\hline$v$ & $0.035 \mathrm{Nm} /(\mathrm{rad} / \mathrm{s}) /$ \\
\hline$I_{s}$ & $0.0713 \mathrm{Kgm}{ }^{2}$ \\
\hline
\end{tabular}

Table 2: Mobile parameter of the WMR P3-DX.

\begin{tabular}{|c|c|}
\hline Parameters & Values \\
\hline$r$ & $0.095 \mathrm{~m}$ \\
\hline$l$ & $0.165 \mathrm{~m}$ \\
\hline$m_{c}$ & $6.04 \mathrm{Kg}$ \\
\hline$m_{\omega}$ & $1.48 \mathrm{Kg}$ \\
\hline
\end{tabular}

\subsection{Energy model}

After analysing all lost components, the total energy model equation is presented as [10]:

$$
E_{\text {battery }}=E_{d c}+E_{k}+E_{f}+E_{\text {elect }}
$$

Where $E_{d c}$ is the energy loss for DC motor, $E_{k}$ is the kinetic energy losses, $E_{f}$ is the energy losses due to friction, $E_{\text {elect }}$ is the energy losses in the electronics, and $E_{\text {battery }}$ is the energy of battery used for the robot.

The kinetic energy of the robot at any time can be expressed as [5]:

$$
E_{k}=\frac{1}{2} m_{c}(v(t))^{2}+\frac{1}{2} I(\omega(t))^{2}
$$

Where $m_{c}$ and $I$ are the mass and the moment of inertia of the mobile.

\subsection{Pioneer mobile robot}

The Pioneer 3DX, shown in Figure 4, has high-speed, high torque, reversible-DC motors, each equipped with encoders for position and speed and advanced deadreckoning. The mobile robot parameters [11] can be obtained from the mobile robot manufacturer data as shown in Tables 1 and 2. Some of the parameters are estimated.

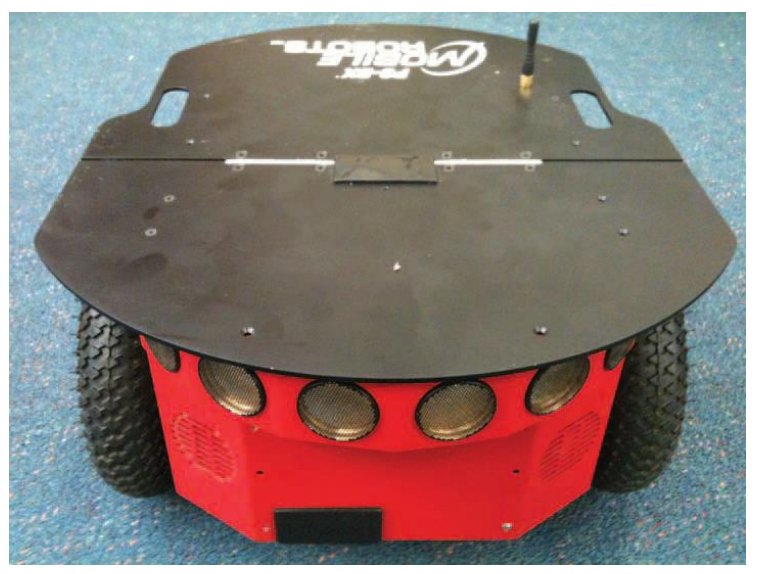

Fig. 4. Pioneer 3DX mobile robot

\section{Model Results}

To Estimate Power and Energy consumption for the P3 DX ,mobile robot, the model displayed in Figure 3 is implemented in MATLAB using Simulink and Simscape tools. The model is simulated in different scenarios as shown in Figure 5, Figure 7, and Figure 9. In the Figures, the green square block represents the start position, the red crosses are the set of points' path which

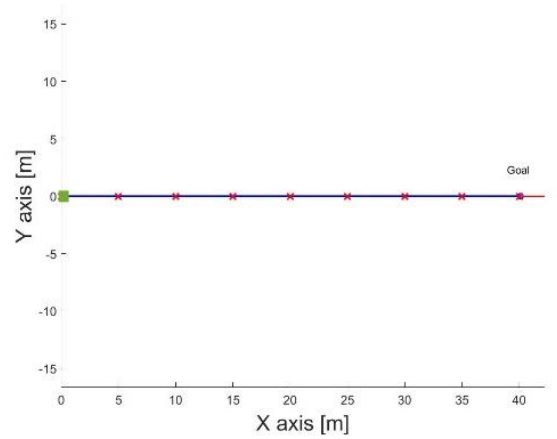

the mobile have to track.

Fig.5 . Scenario 1

During path planning, generally, the robot is considered to be a point mass and represented as a blue circle. Due to the physical dimensions of the robot, the posture and the limitations on the achievable 
velocities/accelerations, the trajectory will get modified as shown in Figure 7 and Figure 9.

In the first scenario, a straightforward path is considered. In this case, the mobile robot is located along the $\mathrm{x}$-axis at $(0,0)$ and tracks the path with the linear reference velocity $v=0.5 \mathrm{~ms}^{-1}$ and the angular reference velocity $\omega=0 \mathrm{rads}^{-1}$.

Our simulation, Figure 6 , shows that the time taken by the mobile robot from the start location to reach the goal location is $80.0 \mathrm{sec}$ and 700 joules are needed to achieve the path.

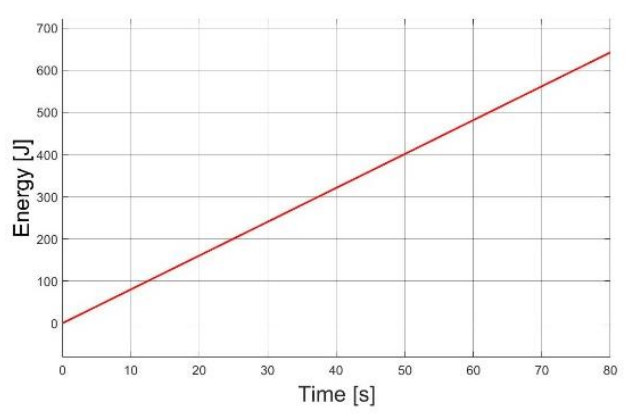

Fig. 6. Energy consumption during scenario 1

The second scenario is a square path with parameters given in Table 3. In order to evaluate the influence of the linear end angular velocities on the tracking path firstly linear reference velocity $v=0.5 \mathrm{~ms}^{-1}$ and the angular reference velocity $\omega=0.1 \mathrm{rads}^{-1}$ limits are considered. Figure 7 shows that in this case the average cross-track error is greater than desired, and 800 Joules are needed to complete the task as shown in Figure 8. With $v=0.05 \mathrm{~ms}^{-1}$ and the angular reference velocity $\omega=0.1 \mathrm{rads}^{-1}$ limits, the mobile flows better the square path and take $786 \mathrm{~s}$ and need 67.5 Joules to archive the path as shown in figures 9 and 10 .

Table 3. Parameters of a square path

\begin{tabular}{|c|c|c|}
\hline Waypoints & $\mathbf{X}$-axis $[\mathbf{m}]$ & Y-axis $[\mathbf{m}]$ \\
\hline 0 & 0 & 0 \\
\hline 1 & 10 & 0 \\
\hline 2 & 10 & 10 \\
\hline 3 & 0 & 10 \\
\hline 4 & 0 & 0 \\
\hline
\end{tabular}

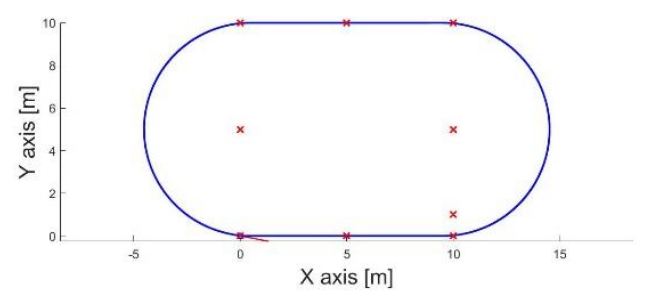

Fig. 7. scenario 2 with $v=0.5 \mathrm{~ms}^{-1}$ and $\omega=0 \mathrm{rads}^{-1}$.

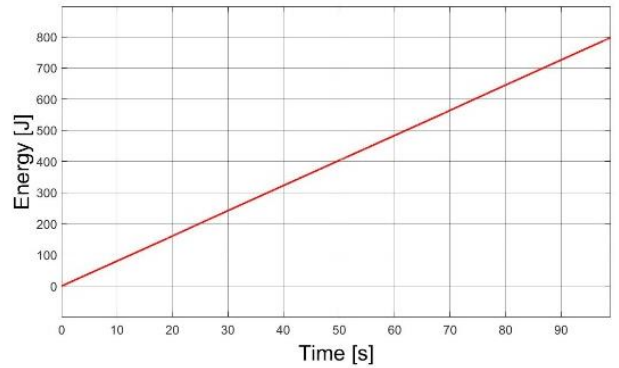

Fig. 8. Energy consumption during scenario 2 with $\mathrm{v}=0.5 \mathrm{~ms}^{-1}$ and $\omega=0 \mathrm{rads}^{-1}$.

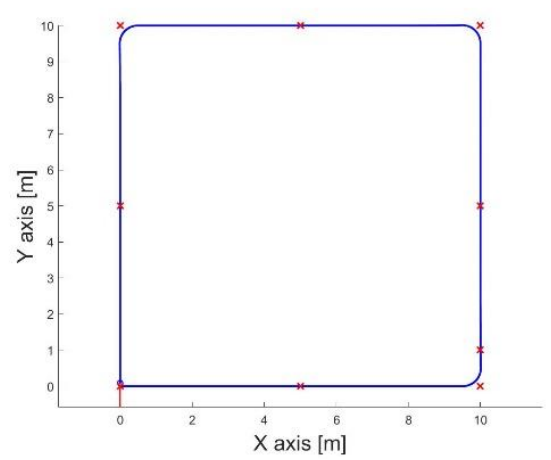

Fig. 9. scenario 2 with $v=0.05 \mathrm{~ms}^{-1}$ and $\omega=0.1 \mathrm{rads}^{-1}$.

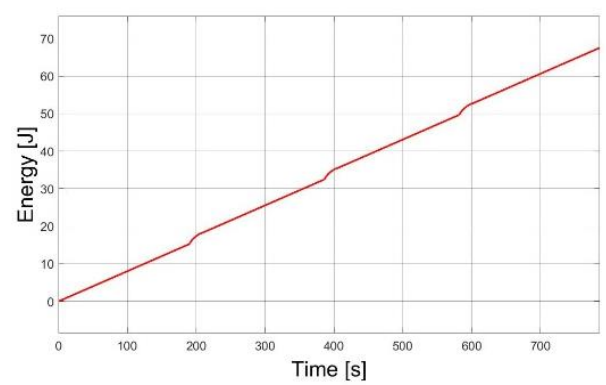

Fig. 10. Energy consumption during scenario 2 with $\mathrm{v}=0.05 \mathrm{~ms}^{-1}$ and $\omega=0.1 \mathrm{rads}^{-1}$.

\section{Conclusion}

In this paper, an estimation of energy consumption for differential guide mobile robot was studied. The simulation results show that there is a trade-off between a using good algorithm tracking path and optimized energy consumption, especially during a complex path. The influence of the parameters as velocities were investigated and discussed to obtain a qualitative tracking path. 


\section{References}

1. H. Kim, B.K. Kim, Minimum-energy translational trajectory generation for differential driven wheeled mobile robots Journal of Intelligent and robotic Systems, 49, pp. 367-383 (2007)

2. H. Kim, B.-K. Kim, Minimum-energy translational trajectory planning for battery-powered threewheeled Omni-directional mobile robots, in Control, Automation, Robotics and Vision, 10thInternational Conference , pp. 17301735, Dec (2008)

3. P. Tokekar, N. Karnard, V. Isler, Energy-Optimal trajectory planning for car-like robots, Autonomous Robots Journal, 37, pp.279, Oct (2014)

4. S. Liu, D. Sun, Optimal motion planning of a mobile robot with minimum energy consumption, presented at the Int. Conf. Advanced Intelligent Mechatronics, Budapest Hungary (2011).

5. S. Liu, D. Sun, Minimizing energy consumption of wheeled mobile robots via optimal motion planning, IEEE/ASME Trans. Mechatronics, 19, pp.401, Apr (2014)

6. Z. Sun, J. Reif, On finding energy-minimizing paths on terrains, Trans. Robotics, Vol. 21, pp.102, Feb (2005)

7. Y. Mei, Y. Lu, Y. Hu, C. Lee, Energy-efficient motion planning for mobile robots, presented at the Int. Conf. Robotics and Automation, New Orleans USA, (2004)

8. M. F. J. Morales and J. B. G. Mendoza, Mixed Energy Model for a Differential Guide Mobile Robot, 23rd International Conference on Methods \& Models in Automation Robotics (MMAR), Miedzyzdroje, pp. 114-119, (2018)

9. Y. Yamamoto, X. Yun, Coordination locomotion and manipulation of a mobile manipulator, IEEE trans. Automatic Control, 39, pp. 1326, Jun (1994)

10. M.Wahab, F.Rios-Gutierrez, A. El Shahat, Energy modeling of differential drive robots, in Proc. SoutheastCon, Fort Lauderale, FL, USA, pp. 1-6Y, (2015)

11. Pioneer 3DX Operational Manual, Mobile Robot Inc., Tech. Rep, (2007) 